\title{
The Explanation of the Michelson-Morley Experiment Results by Means Universal Frame of Reference
}

\author{
Karol Szostek ${ }^{1}$, Roman Szostek ${ }^{2}$ \\ ${ }^{1}$ Dept of Thermodynamics and Fluid Mechanics, Rzeszow University of Technology, Rzeszow, Poland \\ ${ }^{2}$ Department of Quantitative Methods, Rzeszow University of Technology, Rzeszow, Poland \\ Email: kszostek@prz.edu.pl,rszostek@prz.edu.pl
}

How to cite this paper: Szostek, K. and Szostek, R. (2017) The Explanation of the Michelson-Morley Experiment Results by Means Universal Frame of Reference. Journal of Modern Physics, 8, 1868-1883. https://doi.org/10.4236/jmp.2017.811110

Received: August 24, 2017

Accepted: October 24, 2017

Published: October 27, 2017

Copyright @ 2017 by authors and Scientific Research Publishing Inc. This work is licensed under the Creative Commons Attribution International License (CC BY 4.0).

http://creativecommons.org/licenses/by/4.0/

\section{c) (i) Open Access}

\begin{abstract}
It is commonly thought that the Michelson-Morley experiment from 1887 and Kennedy-Thorndike experiment from 1932 demonstrated that the universal frame of reference (ether) does not exist and that the velocity of light in vacuum is absolutely constant. The analysis of this experiment led to the creation of the Special Theory of Relativity (STR). The article explains why Michelson-Morley and Kennedy-Thorndike experiments could not detect the universal frame of reference. In this article, a different transformation of time and position than the Lorentz transformation is derived on the basis of the geometric analysis of the Michelson-Morley and Kennedy-Thorndike experiments. The transformation is derived based on the assumption that the universal frame of reference (UFR) exists. UFR is a frame of reference in which the velocity of light is constant in every direction. In inertial frames of reference moving in the UFR, the velocity of light may be different. The article has derived the formula for relative speed and patterns for the maximum and minimum speed of light that can be measured in the inertial system. Finally, the anisotropy of the microwave background radiation has been explained by using the presented theory. According to the body kinematics model presented in this article, anisotropy of cosmic microwave background is the Doppler effect for observer moving in the UFR.
\end{abstract}

\section{Keywords}

Kinematics of Bodies, Universal Frame of Reference, Coordinate and Time Transformation, One-Way Speed of Light, Anisotropy of Cosmic Microwave Background 


\section{Introduction}

The article an explanation of the results of the Michelson-Morley [1] and Kennedy-Thorndike [2] experiments, assuming the existence of the universal frame of reference (UFR), in which the velocity of light is constant, is presented. In inertial frames of reference moving in the UFR, the velocity of light may be different. The transformations from the inertial system to the UFR and from the UFR to the inertial system were derived by the geometric method.

The velocity of light in one direction has never been accurately measured. In all accurate laboratory experiments, as in the Michelson-Morley experiment, only the average velocity of light, travelling on a closed trajectory, was measured. In these experiments, light always comes back to the source point. Therefore, the assumption about the constant velocity of light (instantaneous velocity) adopted in the Special Theory of Relativity is not experimentally justified. The derivation presented in this article is based on the assumption resulting from these experiments, that is for every observer, the average velocity of light travelling the way to and back is constant.

The transformation "UFR_inertial system" (27)-(28) derived in this article by the geometric method was already derived in articles [3] and [4] by other method. In article [4] the author obtained this transformation from the Lorentz transformation thanks to the synchronization of clocks in inertial frames by the external method. The transformation obtained in the work [4] is the Lorentz transformation differently written down after a change in the manner of time measurement in the inertial frame of reference, this is why the properties of the Special Theory of Relativity were attributed to this transformation. The transformation (27)-(28) has a different physical meaning than the Lorentz transformation, because according to the theory outlined in this article, it is possible to determine the speed with respect to a universal frame of reference by local measurement. So the universal reference system is real, and this is not a freely chosen inertial system.

\section{The Assumptions}

In the presented analysis of the Michelson-Morley and Kennedy-Thorndike experiments, the following assumptions are adopted:

I). There is a universal frame of reference (UFR) with respect to which the velocity of light in vacuum is the same in every direction.

II). The average velocity of light on its way to and back is for every observer independent of the direction of light propagation. This results from the Michelson-Morley experiment.

III). The average velocity of light on its way to and back does not depend on the velocity of the observer in relation to the UFR. This results from the Kennedy-Thorndike experiment.

IV). In the direction perpendicular to the direction of the velocity of the body, moving in relation to the UFR, there is no contraction or elongation of its 
length.

V). The transformation "UFR-inertial system" is linear.

The transformation derivation presented in this article differs from the derivation by the geometric method of the Lorentz transformation which is the basis for the STR. In STR in the derivation of the Lorentz transformation, it is assumed that the reverse transformation has the same form as the original transformation. Such an assumption stems from the belief that all inertial frames are equivalent. In the derivation presented in this article, we do not assume what form the reverse transformation has.

Assumptions concerning the velocity of light adopted in this article are also weaker than those adopted in the STR. In the STR, it is assumed that the velocity of light is absolutely constant, despite the fact that it has not been proven by any experiment. In this article, the assumption resulting from experiments is adopted, i.e. the average velocity of light on the way to the mirror and back is constant (assumption II and III). In the presented considerations, the velocity of light by assumption is constant only in one highlighted frame of reference-the UFR (assumption I).

Assumptions IV and V are identical to those on which the STR is based.

In works [5] and [6], identical transformations were derived as in this article, but with the adopted additional assumption. For this, it was necessary to conduct the full analysis of the Michelson-Morley experiment in which also the second stream of light, parallel to velocity $\mathrm{v}$, is taken into account. In that case, only one stream of light was analyzed.

\section{Time and Way of the Light Flow in the UFR}

Let us consider inertial system $U^{\prime}$, which moves in relation to system $U$ related with the UFR at velocity $v$ (Figure 1 ). In system $U^{\prime}$, there is a mirror at distance $D^{\prime}$ from the beginning of the system. Light in the system $U$ moves at constant velocity $c$. From system $U^{\prime}$, from point $x^{\prime}=0$ in time $t=0$, a stream of light was sent in the direction of the mirror. Having reached the mirror, the reflected light moves in the system $U$ in the opposite direction at velocity with the negative value $-c$.

We assume the following symbols for the observer from the system $U: t_{1}$ is the

(a)

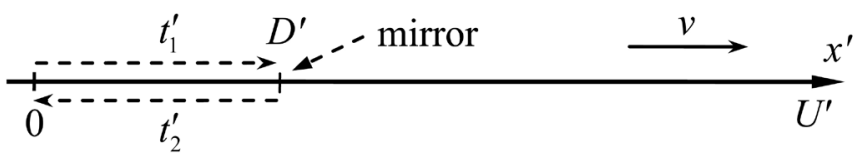

(b)

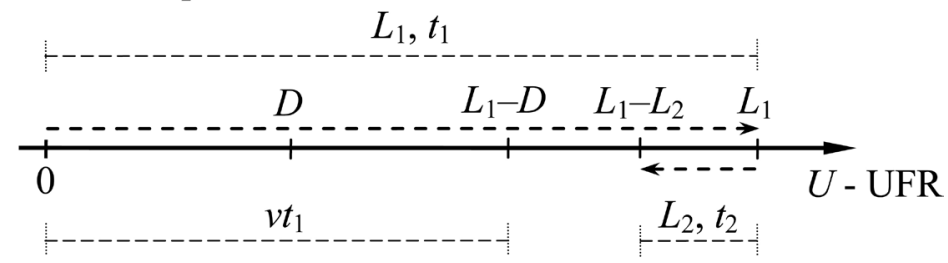

Figure 1. The time and the flow path of light to the mirror and back: (a) the way of light seen from the inertial system $U^{\prime},(b)$ the way of light seen from the UFR. 
time of the light flow to the mirror, $t_{2}$ is the time of the light return to the starting point. $L_{1}$ and $L_{2}$ are ways which were travelled by light in the system $U$ in one direction and in another.

When light moves in the direction of the mirror, then the mirror runs away from it at velocity $v$. When light comes back to point $x^{\prime}=0$ after the reflection from the mirror, then this point runs towards it at velocity $v$. For an observer from system $U$, distance $D^{\prime}$ parallel to velocity vector $v$ is seen as $D$. We obtain

$$
\begin{gathered}
L_{1}=D+v \cdot t_{1}, \quad L_{2}=D-v \cdot t_{2} \\
t_{1}=\frac{L_{1}}{c}=\frac{D+v \cdot t_{1}}{c}, \quad t_{2}=\frac{L_{2}}{c}=\frac{D-v \cdot t_{2}}{c}
\end{gathered}
$$

Dependencies (2) should be solved due to $t_{1}$ and $t_{2}$. We then obtain time and way of flow in the UFR

$$
\begin{gathered}
t_{1}=\frac{D}{c-v}, \quad t_{2}=\frac{D}{c+v} \\
L_{1}=c \cdot t_{1}=D \frac{c}{c-v}, \quad L_{2}=c \cdot t_{2}=D \frac{c}{c+v}
\end{gathered}
$$

\section{The Geometrical Derivation of the Transformation}

We analyze the results of the Michelson-Morley experiment, as shown in Figure 2. The inertial system $U^{\prime}$ move sat a relative velocity $v$ to the inertial system $U$,
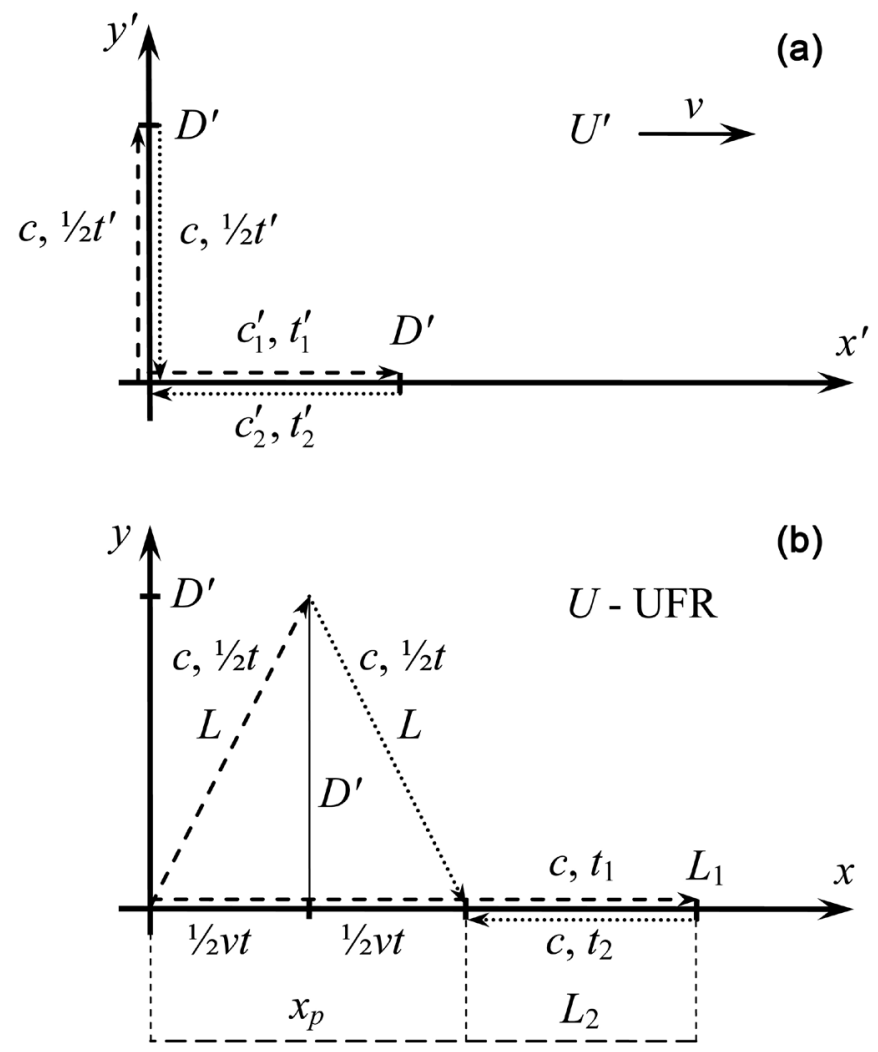

Figure 2. Paths of two streams of light: (a) seen by an observer from the system $U^{\prime}(b)$ seen by an observer from the system $U$ (UFR). 
associated with the UFR, parallel to the axis $x$. Axes $x$ and $x^{\prime}$ lie on one straight line.

At the moment when origins of systems overlap, clocks in both systems are synchronized. Clocks in system $U$ related to the UFR are synchronized by the internal method [4]. Clocks in system $U^{\prime}$ are synchronized by the external method in such a manner that if the clock of system $U$ indicates time $t=0$, then the clock of system $U^{\prime}$ next to it is also reset, that is $t^{\prime}=0$.

In the system $U^{\prime}$, an experiment measuring the velocity of light in vacuum perpendicular and parallel to the direction of movement of the system $U^{\prime}$ in relation to the UFR was conducted. In each of these directions, light travels to the mirror and back. Figure 2 presents in part (a) the flow path of light seen by the observer from the system $U^{\prime}$, while in part (b) the path seen by the observer from the system $U$.

In system $U$ light has always constant velocity $c$ (assumption I). Considerations concern the flow of light in vacuum.

In accordance with conclusions resulting from the Michelson-Morley experiment it has been assumed that the average velocity of light $c_{p}$ on the way to the mirror and back in system $U^{\prime}$ is the same in every direction, in particular in the parallel direction to the axis $y^{\prime}$ (assumption II). It has also been assumed that the average velocity of light $c_{p}$ on the way to the mirror and back does not depend on the velocity of an observer in relation to the UFR (assumption III).

From assumption II and III it follows that the average velocity of light $c_{p}$ in the inertial frame of reference is the same as the velocity of light $c$ in the system $U$. If we allow that the average velocity of light $c_{p}$ in the system $U^{\prime}$ is a function of the velocity of light $c$ in the system $U$ dependent on the velocity $v$, we can write

$$
c_{p}=f(v) c
$$

From assumption III the average velocity of light is the same for different velocities of the Earth relative to the UFR, so $f\left(v_{1}\right)=f\left(v_{2}\right)$. Since $f(0)=1$, therefore $f(v)=1$ for every velocity $v$. It follows that $c=c_{p}$.

The mirrors are associated with the system $U^{\prime}$ and placed at distance $D^{\prime}$ from the origin. One mirror is located on the axis $x^{\prime}$, the second one on the axis $y^{\prime}$. We assume that the distance $D^{\prime}$, which is perpendicular to the velocity $v$ is the same for observers from both systems (assumption IV). Therefore, in Figure 2, there is the same length $D^{\prime}$ in part $a$ ) and part $b$ ).

The flow time of light in the system $U$, along the axis $x$, in the direction to the mirror is marked as $t_{1}$. The flow time back is marked as $t_{2}$.

The flow time of light in the system $U^{\prime}$, along the axis $x^{\prime}$, in the direction to the mirror is marked as $t_{1}^{\prime}$. The flow time back to the source is marked $t_{2}^{\prime}$.

Total time is marked respectively as $t$ and $t^{\prime}\left(t=t_{1}+t_{2}\right.$ and $\left.t^{\prime}=t_{1}^{\prime}+t_{2}^{\prime}\right)$.

The light stream, moving parallel to the axis $y^{\prime}$, from the point of view of the system $U$ moves along the arms of an isosceles triangle of side length $L$. Since the velocity of light is constant in the system $U$, therefore, the time of movement along both arms is the same and is equal to $t / 2$. 
In the system $U$, the light stream parallel to the axis $x$, in the direction of the mirror overcomes distance $L_{1}$ during time $t_{1}$. On the way back, it travels distance $L_{2}$ during time $t_{2}$. These distances are different due to the movement of the mirror and the source point of light in the UFR.

In the experiment, both light streams come back to the source point at the same time, both in system $U$ and system $U$ ' It results from assumption II and from the mirrors setting at the same distance from the point of light emission.

For an observer of $U^{\prime}$ and $U$, the velocity of light can be written as

$$
\frac{2 D^{\prime}}{t_{1}^{\prime}+t_{2}^{\prime}}=\frac{2 D^{\prime}}{t^{\prime}}=c=\frac{2 L}{t}=\frac{L_{1}+L_{2}}{t_{1}+t_{2}}
$$

From Equation (6) light paths $L$ and $D^{\prime}$ as a function of the velocity of light $c$ and the light flow times $t, t^{\prime}$ respectively in the systems $U$ and $U^{\prime}$ can be determined

$$
L=\frac{c t}{2} ; \quad D^{\prime}=\frac{c t^{\prime}}{2}
$$

The velocity of the system $U^{\prime}$ relative to the absolute frame of reference $U$, i.e. the UFR is marked by $v$. Since $x_{p}$ is the path that the system $U^{\prime}$ travelled in time $t$, of the light flow, we have

$$
v=\frac{x_{p}}{t} ; \quad x_{p}=v t
$$

Using the geometry of Figure 2, the length $L$ can be expressed as

$$
L=\sqrt{\left(x_{p}\right)^{2}+D^{\prime 2}}=\sqrt{(v t / 2)^{2}+D^{\prime 2}}
$$

Having squared Equation (9) and taken (7) into account, we obtain

$$
(c t / 2)^{2}=(v t / 2)^{2}+\left(c t^{\prime} / 2\right)^{2}
$$

After arranging we obtain

$$
\begin{gathered}
t^{2}\left(c^{2}-v^{2}\right)=\left(c t^{\prime}\right)^{2} \\
t=t^{\prime} \frac{1}{\sqrt{1-(v / c)^{2}}} \text { for } x^{\prime}=0
\end{gathered}
$$

The above relation describes only times $t$ and $t^{\prime}$ that involve the full light flow to the mirror and back. It should be noted that these are times measured in point $x^{\prime}=0$. However, if we assume that the length $D^{\prime}$ can be chosen so that time flow of light is any time, so the relationship (12) is true for any time.

Length $D^{\prime}$ associated with the system $U^{\prime}$ that is parallel to the axis $x$, and is seen from the system $U$ as $D$. If light flows in the absolute frame of reference $U$ to the mirror, is chasing the mirror, which is away from it at length $D$. After reflection, light returns to the source point, which runs against him. Using Equations (4), we obtain the equations for light flow paths in both directions along the axis $x^{\prime}$ in the system $U$

$$
L_{1}=c t_{1}=D \frac{c}{c-v} ; \quad L_{2}=c t_{2}=D \frac{c}{c+v}
$$


From Equation (13) the sum and difference in length the $L_{1}$ and $L_{2}$, which light travelled in the system $U$, can be determined

$$
\begin{aligned}
& L_{1}+L_{2}=D \frac{c}{c-v}+D \frac{c}{c+v}=2 D \frac{1}{1-(v / c)^{2}}, \\
& L_{1}-L_{2}=D \frac{c}{c-v}+D \frac{c}{c+v}=2 D \frac{v}{c} \cdot \frac{1}{1-(v / c)^{2}}
\end{aligned}
$$

From the second equation, the distance that the system $U^{\prime}$ travelled in half of the light flow time $t / 2$ can be determined, so we have

$$
x_{p} / 2=v t / 2=\frac{L_{1}-L_{2}}{2}=D \frac{v}{c} \cdot \frac{1}{1-(v / c)^{2}}
$$

Since it was assumed that in the system $U$ the velocity of light $c$ is constant, therefore both distances, which are travelled by light $2 L$ and $L_{1}+L_{2}$ are the same

$$
2 L=L_{1}+L_{2}
$$

After substituting (9) and the first Equation (14) we obtain

$$
2 \sqrt{(v t / 2)^{2}+D^{\prime 2}}=2 D \frac{1}{1-(v / c)^{2}}
$$

After reducing by two, raising to the square and taking (15) into account we can write

$$
\left(D \frac{v}{c} \cdot \frac{1}{1-(v / c)^{2}}\right)^{2}+D^{\prime 2}=D^{2}\left(\frac{1}{1-(v / c)^{2}}\right)^{2}
$$

From Equation (18) a dependence for the length contraction can be determined

$$
\begin{gathered}
D^{\prime 2}=D^{2}\left(\frac{1}{1-(v / c)^{2}}\right)^{2}\left(1-(v / c)^{2}\right) \\
D^{\prime}=D\left(\frac{1}{1-(v / c)^{2}}\right) \sqrt{1-(v / c)^{2}}=D \frac{1}{\sqrt{1-(v / c)^{2}}} \\
D=D^{\prime} \sqrt{1-(v / c)^{2}}
\end{gathered}
$$

Lengths $D$ and $D^{\prime}$ which are distances between mirrors and the point of light emission occur in the above dependence. Since length $D^{\prime}$ can be selected on a voluntary basis; therefore, dependence (20) is true for any value of $D^{\prime}$.

Having introduced (12) to (8), we have

$$
x_{p}=v t^{\prime} \frac{1}{\sqrt{1-(v / c)^{2}}} \text { for } x^{\prime}=0
$$

We assume that the transformation from the inertial system $U^{\prime}$ to the system $U$ is linear (assumption $\mathrm{V}$ ). If linear factors dependent on $x^{\prime}$ are added to the transformation of time and position (12), (21), transformations with unknown coefficients $a, b$ can be obtained 


$$
\begin{aligned}
& t=t^{\prime} \frac{1}{\sqrt{1-(v / c)^{2}}}+a x^{\prime} \\
& x=v t^{\prime} \frac{1}{\sqrt{1-(v / c)^{2}}}+b x^{\prime}
\end{aligned}
$$

Transformation (22) should be valid for any time and position. In a particular case, it is valid at the moment of clocks' synchronization, that is when $t=t^{\prime}=0$ for the point with coordinates $D^{\prime}$ in system $U^{\prime}$. In this connection, we introduce $t$ $=t^{\prime}=0, x^{\prime}=D^{\prime}$ and $x=D$ into (22). At this point it has been applied external synchronization of clocks in a $U^{\prime}$ on the basis of clocks in UFR. Having taken (20) into account, we obtain

$$
\begin{aligned}
& 0=a D^{\prime} \\
& \sqrt{1-(v / c)^{2}} D^{\prime}=b D^{\prime}
\end{aligned}
$$

We obtain coefficients $a$ and $b$

$$
\begin{aligned}
& a=0 \\
& b=\sqrt{1-(v / c)^{2}}
\end{aligned}
$$

Finally, the transformation from any inertial system $U^{\prime}$ to the system $U$, associated with the UFR takes the form

$$
\begin{gathered}
t=\frac{1}{\sqrt{1-(v / c)^{2}}} t^{\prime} \\
x=\frac{1}{\sqrt{1-(v / c)^{2}}} v t^{\prime}+\sqrt{1-(v / c)^{2}} \cdot x^{\prime}
\end{gathered}
$$

After transformations of the above equations, we obtain the inverse transformation, that is the transformation from the system $U$, associated with the UFR to the inertial system $U^{\prime}$

$$
\begin{gathered}
t^{\prime}=\sqrt{1-(v / c)^{2}} \cdot t \\
x^{\prime}=\frac{1}{\sqrt{1-(v / c)^{2}}}(-v t+x)
\end{gathered}
$$

\section{Relative Velocities between Systems}

We will now denote inertial system $U^{\prime}$ as $U_{1}$. From this inertial system, another inertial system $U_{2}$ is observed. In relation to the system $U$ (UFR), inertial system $U_{1}$ has velocity $v_{1}$, while inertial system $U_{2}$ has velocity $V_{2}$. We determine the relative velocity $V_{2 / 1}$ of system $U_{2}$ seen from system $U_{1}$.

Let $\mathrm{d} x$ be a change in the position of system $U_{2}$ in time $\mathrm{d} t$ seen from system $U$. Now it can be written that

$$
v_{2}=\frac{\mathrm{d} x}{\mathrm{~d} t}
$$

Let $\mathrm{d} x_{1}$ be a change in the position of system $U_{2}$ in time $d t_{1}$ seen from system 
$U_{1}$. Now it can be written that

$$
v_{2 / 1}=\frac{\mathrm{d} x_{1}}{\mathrm{~d} t_{1}}
$$

In order to determine the relative velocity of system $U_{2}$ in relation to system $U_{1}$, we will calculate differentials from transformations (27)-(28) $\left(t_{1}=t^{\prime}, x_{1}=x^{\prime}\right.$, $\left.v_{1}=v\right)$

$$
\left\{\begin{array}{l}
\mathrm{d} t_{1}=\sqrt{1-\left(v_{1} / c\right)^{2}} \cdot d t \\
\mathrm{~d} x_{1}=\frac{1}{\sqrt{1-\left(v_{1} / c\right)^{2}}}\left(-v_{1} \mathrm{~d} t+\mathrm{d} x\right)
\end{array}\right.
$$

We introduce the above differentials into formula (30)

$$
\begin{gathered}
v_{2 / 1}=\frac{\frac{1}{\sqrt{1-\left(v_{1} / c\right)^{2}}}\left(-v_{1} \mathrm{~d} t+\mathrm{d} x\right)}{\sqrt{1-\left(v_{1} / c\right)^{2}} \mathrm{~d} t} \\
v_{2 / 1}=\frac{-v_{1}+\frac{\mathrm{d} x}{\mathrm{~d} t}}{1-\left(v_{1} / c\right)^{2}}
\end{gathered}
$$

Having taken dependence (29) into account, we obtain the searched formula for the relative velocity of inertial system $U_{2}$ in relation to inertial system $U_{1}$

$$
v_{2 / 1}=\frac{v_{2}-v_{1}}{1-\left(v_{1} / c\right)^{2}}
$$

\section{The Velocity of Light in the Inertial Frame of Reference}

Now we calculate the velocity of light in any inertial system $U_{1}$.

Consider three inertial reference systems $U_{1}, U_{2}$ and $U_{3}$ moving in the UFR in parallel to the $x$ axis, Figure 3 .

Systems $U_{2}$ and $U_{3}$ are associated with light, but move in opposite directions with velocities light in the vacuum $c_{p 1}=v_{2 / 1}$ and $c_{p 2}=V_{3 / 1}$ in the system $U_{1}$. Therefore, their velocities in the UFR are $v_{2}=c$ and $v_{3}=-c$. The system $U_{1}$ moves in the UFR at the velocity $v_{1} \geq 0$. From equation (34), the velocity of light. measured in the system $U_{1}$, can be calculated

$$
c_{p 1}=v_{2 / 1}=\frac{c-v_{1}}{1-\left(v_{1} / c\right)^{2}}=\frac{c^{2}\left(c-v_{1}\right)}{c^{2}-v_{1}^{2}}=\frac{c^{2}\left(c-v_{1}\right)}{\left(c+v_{1}\right)\left(c-v_{1}\right)}=\frac{c^{2}}{c+v_{1}} \leq c
$$

and

$$
c_{p 2}=v_{3 / 1}=\frac{-c-v_{1}}{1-\left(v_{1} / c\right)^{2}}=-\frac{c^{2}\left(c+v_{1}\right)}{c^{2}-v_{1}^{2}}=-\frac{c^{2}\left(c+v_{1}\right)}{\left(c+v_{1}\right)\left(c-v_{1}\right)}=-\frac{c^{2}}{c-v_{1}} \leq-c
$$

When light travels in the UFR in the same direction as the inertial system $U_{1}$, then the velocity of light in the system is expressed by Equation (35). When light travels in the UFR in the direction opposite to the inertial system $U_{1}$, then the 


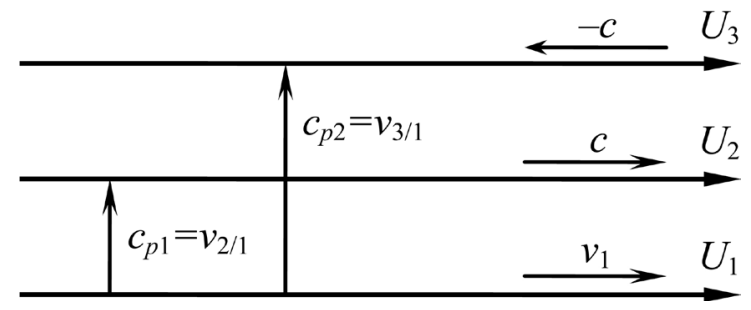

Figure 3. The velocity of light in one direction.

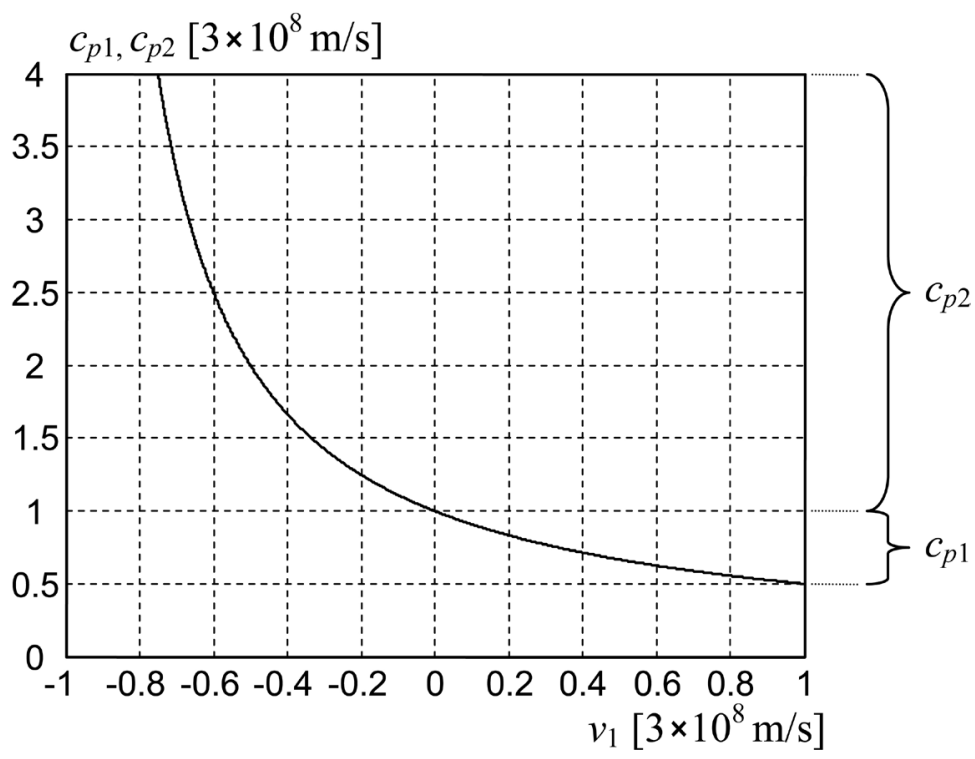

Figure 4. The velocity of light in the inertial system moving with the speed $v_{1}$ relative to the UFR.

velocity of light in the system is expressed by Equation (36). The velocity of light in system $U_{1}$ assumes values as in Figure 4.

It follows that if the system $U_{1}$ moves at velocity close to $c$, then light running in the same direction in relation to the system $U_{1}$ has velocity close to $c / 2$. Light running in the opposite direction has infinite velocity to the system $U_{1}$. Thus, the velocity of light in relation to the inertial system can be very high, because the system clocks go more slowly than in the UFR. The velocity of light in the UFR is exactly $c$.

In the system $U_{1}$, let light run in parallel to velocity $v_{1}$ of the system $U_{1}$ relative to the UFR. Just as in the Michelson-Morley experiment, light runs along the way $L$ over time $t^{\prime}$. At the end of the way, light is reflected in the mirror and goes back along the same way $L$ over time $t^{\prime \prime}$. Then, the average velocity of light can be described on the basis of (35) and (36) and is equal $c$.

This velocity agrees with the results of the Michelson-Morley and Kennedy-Thorndike experiments, which shows that the average velocity of light is constant and is equal to $c$ (the average velocity, not instantaneous). We have shown that the Michelson-Morley experiment does not imply that the current velocity of light is constant in every direction. 
Velocities expressed in dependencies (35) and (36) are different. The first one refers to the direction compatible with velocity $V_{1}$, and the other concerns the direction opposite to velocity $v_{1}$. However, the average velocity of light is constant and is equal to $c$.

In works [7] and [8] a general formula for the velocity of light $c_{\alpha^{\prime}}^{\prime}$ running in any direction in vacuum is derived. It has the form of

$$
c_{\alpha^{\prime}}^{\prime}=\frac{c^{2}}{c+v \cos \alpha^{\prime}}
$$

For light moving in a material medium motionless in relation to the observer, it has the form of [7] ( $c_{\alpha^{\prime}}^{\prime}$ is a one-way light velocity in a material medium moving together with the observer)

$$
c_{s \alpha^{\prime}}^{\prime}=\frac{c^{2} c_{s}}{c^{2}+c_{s} v \cos \alpha^{\prime}}
$$

In these two dependencies, angle $\alpha^{\prime}$ is the angle, measured by the observer, between the vector of its velocity in relation to the UFR and the vector of the velocity of light. Velocity $c_{s}$ is the velocity of light in the material medium motionless in relation to the UFR seen by the observer motionless in relation to the UFR.

Formulas (37) and (38) come down to formulas (35) and (36), if we substitute $c_{s}=c$ and $\alpha^{\prime}=0 \mathrm{rad}$ or $\alpha^{\prime}=\pi \mathrm{rad}$. For the velocity of light expressed by formula (38), the average velocity on the way to the mirror and back is equal to

$$
\begin{aligned}
& c_{s r}^{\prime}=\frac{2 L}{t_{s \alpha^{\prime}}^{\prime}+t_{s\left(\pi-\alpha^{\prime}\right)}^{\prime}}=\frac{\frac{2 L}{\frac{c^{2} c_{s}}{c^{2}+c_{s} v \cos \alpha^{\prime}}}+\frac{L}{\frac{c^{2} c_{s}}{c^{2}+c_{s} v \cos \left(\pi-\alpha^{\prime}\right)}}}{2} \\
& c_{s r}^{\prime}=\frac{2}{\frac{c^{2}+c_{s} v \cos \alpha^{\prime}}{c^{2} c_{s}}+\frac{c^{2}-c_{s} v \cos \alpha^{\prime}}{c^{2} c_{s}}}=\frac{2}{\frac{2 c^{2}}{c^{2} c_{s}}}=c_{s}
\end{aligned}
$$

From dependence (40) it follows that $c_{s}$ is also the average velocity of light on the way to the mirror and back in the material medium motionless in relation to the a moving observer. Despite the fact that the velocity of light expressed by formula (38) depends on angle $\alpha^{\prime}$ and velocity $v$, the average velocity of light on the way to the mirror and back is always constant and is equal to $c_{s}$.

\section{Anisotropy of Cosmic Microwave Background}

Light is a special case of electromagnetic radiation, however, the above considerations concern not only light, but each electromagnetic radiation.

The outer space is filled with the microwave background radiation. Numerous studies dedicated to this topic have been discussed in this work [9]. The spectrum of this radiation is the same as the spectrum of the black-body radiation with a temperature of

$$
\bar{T}_{v}=2.726 \pm 0.010 \mathrm{~K}
$$


The microwave background radiation is electromagnetic radiation with a maximum intensity for the frequency of approximately $300 \mathrm{GHz}$. The background radiation has an irregularity (anisotropy) with an amplitude of

$$
\Delta T_{v}=3.358 \pm 0.017 \mathrm{mK}
$$

The lowest temperature of the background radiation can be observed in the vicinity of the Aquarius constellation, while the highest temperature in the vicinity of the Lion constellation.

Attempts have been made to provide numerous explanations for the anisotropy of the microwave background radiation but, in fact, it is evidence confirming the existence of UFR, that is a frame of reference in which the light travels. Anisotropy is caused by the Doppler effect seen by an observer who is moving in relation to the UFR. On that basis, one can determine the velocity with which the Solar System moves in relation to the UFR.

It is a well-known fact that the microwave background radiation passes through matter filling the space easily, and in consequence, if its sources are weak and dispersed, in the long course of the evolution of the universe, this radiation has accumulated evenly in the whole outer space. Therefore, we can assume that the microwave background radiation is homogeneous in the universal frame of reference, and corresponds to the temperature of a black body $T_{0}$.

The work [7] have revealed that on the basis of transformation (25)-(28) it is possible to derive the Doppler's effect equation from the UFR into an inertial frame, the same as it exists in the STW, that is

$$
f_{v}=f_{0} \frac{c-v \cos \alpha_{E}}{\sqrt{c^{2}-v^{2}}} ; \quad \alpha_{E} \in(0 \div \pi)
$$

where: $f_{V}$ is a frequency of the microwave background radiation observed from the Solar System, $f_{0}$ is a frequency of the microwave background radiation seen with the UFR, while $\alpha_{E}$ is an angle between the velocity vector $v$ and the vector of the speed of light. The angle $\alpha_{E}$ is seen from the UFR.

For $\alpha_{E}=0$ the Equation (43) comes down to

$$
f_{v}^{\min }=f_{0} \sqrt{\frac{(c-v)^{2}}{(c+v)(c-v)}}=f_{0} \sqrt{\frac{c-v}{c+v}} \text { for } \alpha_{E}=0
$$

On the basis of the Wien's displacement law, the length of a light wave with a maximum intensity is connected with a temperature of a black body emitting it as presented by this relation

$$
\frac{1}{\lambda_{\max }}=\frac{T}{0.0029[\mathrm{~m} \cdot \mathrm{K}]} \Rightarrow f=\frac{c}{\lambda_{\max }}=\frac{c T}{0.0029}
$$

For the frequency seen in the UFR we get

$$
f_{0}=\frac{c T_{0}}{0.0029}
$$

For the frequency seen by the moving observer 


$$
f_{v}^{\min }=\frac{c T_{v}^{\min }}{0.0029}=\frac{c\left(\bar{T}_{v}-\Delta T_{v}\right)}{0.0029} \text { for } \alpha_{E}=0
$$

After substituting to (44) we receive

$$
T_{v}^{\min }=\bar{T}_{v}-\Delta T_{v}=T_{0} \sqrt{\frac{c-v}{c+v}} \quad \text { for } \alpha_{E}=0
$$

On this basis of

$$
\left(T_{0} \approx \bar{T}_{v} \wedge \alpha_{E}=0\right) \Rightarrow v \approx c \frac{\bar{T}_{v}^{2}-\left(\bar{T}_{v}-\Delta T_{v}\right)^{2}}{\bar{T}_{v}^{2}+\left(\bar{T}_{v}-\Delta T_{v}\right)^{2}}
$$

Finally, similarly to the work [9], we receive the velocity of the Solar System in relation to the UFR $(c=299,792.458 \mathrm{~km} / \mathrm{s})$

$$
v=369.5 \pm 3 \mathrm{~km} / \mathrm{s} \approx 0.001233 \cdot c
$$

This velocity is turned in the direction of the Lion constellation, which corresponds to direction of the galactic coordinates (Figure 5)

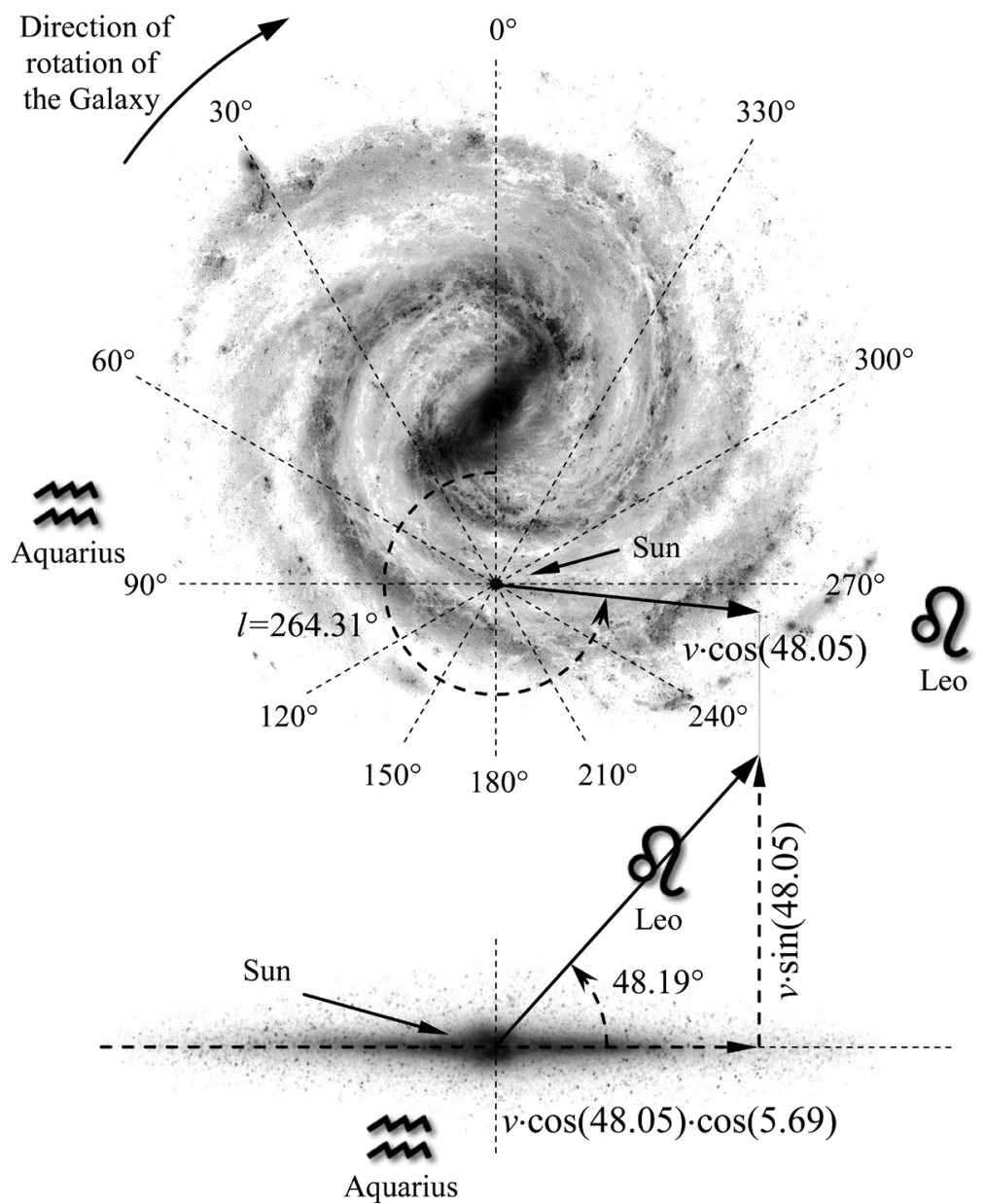

Figure 5. The speed of the Solar System relative to UFR. The projection on the plane of the Galaxy and project on the plane perpendicular to the plane of the Galaxy $\left(90^{\circ}\right.$ $270^{\circ}$ ). View of galaxy Milky Way from above (with marked galactic coordinates) and side view. 


$$
\begin{aligned}
& l=264.31^{\circ} \pm 0.16^{\circ} \\
& b=48.05^{\circ} \pm 0.10^{\circ}
\end{aligned}
$$

Since the measurements of the microwave background radiation were very precise the value of the velocity of the Solar System in relation to the UFR can be regarded as precise. It is interesting that this velocity is of the same order of magnitude as its detrimental estimation of $445 \mathrm{~km} / \mathrm{s}$, calculated on the basis of the experiment with mesons $K^{\dagger}$ presented in the work [7].

\section{Conclusions}

Derived transformations (25)-(26) and (27)-(28) are consistent with the Michelson-Morley and Kennedy-Thorndike experiments. From the above transformations it follows that the measurement of the velocity of light in vacuum by means of the previously applied methods will always give the average value equal to $c$. This happens despite the fact that for the moving observer the velocity of light has a different value in different directions. The average velocity of light is always constant and independent of the velocity of the inertial frame of reference. Due to this property of the velocity of light, the Michelson-Morley and Kennedy-Thorndike experiments could not detect the universal frame of reference.

The adopted assumptions I-V allow for the explanation of the anisotropy of the cosmic microwave background. This anisotropy is caused by the Doppler effect, which results from the movement of the Solar System in relation to the UFR.

It follows from the conducted analysis that the explanation of the results of the Michelson-Morley experiment on the basis of the universal frame of reference is possible. Stating that the Michelson-Morley experiment proved that the velocity of light is absolutely constant is untrue. Stating that the Michelson-Morley experiment proved that there is no universal frame of reference in which light propagates and moves at a constant velocity is also untrue.

Admitting that the velocity of light may depend on the direction of its emission does not differentiate any direction in space. The velocity of light which is measured by the moving observer is significant here. It is the velocity at which the observer moves in relation to the universal frame of reference that differentiates the characteristic direction in space, but only for this observer. For the observer motionless in relation to the universal frame of reference, the velocity of light is always constant and does not depend on the direction of its emission. If the observer moves in relation to the universal frame of reference, then from his perspective space is not symmetrical. The case of this observer will be similar to the case of the observer moving on water and measuring the velocity of the wave on water. Despite that the wave propagates on water at the constant velocity in every direction, from the perspective of the observer moving on water, the velocity of the wave will be different in different directions.

At present, it is believed that the STR is the only theory explaining the Michelson-Morley and Kennedy-Thorndike experiments. This article proved that dif- 
ferent theories in accordance with these experiments are possible. In works [7] [8] and [10], a new physical theory of kinematics and dynamics of bodies based on the transformation determined here, called by the authors the Special Theory of Ether, was derived. In work [8] it has been shown that it is possible to weaken the assumption IV and derive a more general form of transformation (25)-(28). Thus many kinematics can be derived in accordance with the Michelson-Morley and Kennedy-Thorndike experiments. In the work [7] has been shown that within each such kinematics can derive infinitely many dynamics. In order to derive dynamics, it is necessary to adopt the additional assumption, which will allow for introduction into theory of the concepts of mass, kinetic energy, and momentum.

The Michelson-Morley experiment and Kennedy-Thorndike experiment were conducted many times by different teams. Also modified and improved versions of this experiment, like the experiment with sapphire crystals from 2015 [11], were conducted. Each of the experiments only confirmed that the average velocity of light is constant. Therefore, assumptions, on which the presented derivation is based, are experimentally justified.

\section{References}

[1] Michelson, A.A. and Morley, E.W. (1887) American Journal of Science, 34, 333-345. https://doi.org/10.2475/ajs.s3-34.203.333

[2] Kennedy, R.J. and Thorndike, E.M. (1932) Physical Review, 42, 400-418 https://doi.org/10.1103/PhysRev.42.400

[3] Tangherlini, F.R. (1958) The Velocity of Light in Uniformly Moving Frame. A Dissertation, Stanford University, Stanford, CA. (2009) The Abraham Zelmanov Journal, 2, ISSN 1654-9163. http://zelmanov.ptep-online.com/papers/zj-2009-03.pdf

[4] Mansouri, R. and Sexl, R.U. (1977) General Relativity and Gravitation, 8, 497-513. https://doi.org/10.1007/BF00762634

[5] Szostek, K. and Szostek, R. (2016) IOSR Journal of Applied Physics (IOSR-JAP), 8, Version III, 22-30, ISSN 2278-4861. https://doi.org/10.9790/4861-0804032230

[6] Szostek, K. and Szostek, R. (2017) Ученые Записки Физического Факультета МГУ [Memoirs of the Faculty of Physics Lomonosov Moscow State University], 2, 172102, ISSN 2307-9665. http://uzmu.phys.msu.ru/toc/2017/2

[7] Szostek, K. and Szostek, R. (2015) Szczególna Teoria Eteru. [Special Theory of Ether.] Wydawnictwo Amelia, Rzeszów, Polska, ISBN 978-83-63359-77-5. Publishing House Amelia, Rzeszow, Poland, ISBN 978-83-63359-81-2.

http://www.ste.com.pl/

[8] Szostek, K. and Szostek, R. (2017) Wyprowadzenie ogólnej postaci kinematyki z uniwersalnym układem odniesienia. [The Derivation of the General Form of Kinematics with the Universal Reference System.] viXra,

http://www.vixra.org/abs/1704.0105

http://www.vixra.org/abs/1704.0104

[9] Smoot, G.F. (2007) Reviews of Modern Physics, 79, 1349-1379. (2008) Postepy Fizyki, 59, 52-79. (2007) Успехи Физических Наук, 177, 1294-1317.

https://doi.org/10.1103/RevModPhys.79.1349

http://pf.ptf.net.pl/PF-2008-2/docs/PF-2008-2.pdf 
https://doi.org/10.3367/UFNr.0177.200712d.1294

[10] Szostek, K. and Szostek, R. Wyjaśnienie wyników eksperymentu Michelsona-Morleya przy pomocy teorii $z$ eterem. [The Explanation of the Michelson-Morley Experiment Results by Means of the Theory of Ether.] viXra,

http://www.vixra.org/abs/1612.0019 (2016)

http://www.vixra.org/abs/1704.0302 (2017)

[11] Nagel, M., Parker, S.R., Kovalchuk, E.V., Stanwix, P.L., Hartnett, J.G., Ivanov, E.N., Peters, A. and Tobar, M.E. (2015) Nature Communications, 6, Article Number: 8174. https://doi.org/10.1038/ncomms9174 